\title{
Escala de Autoeficácia no Trabalho: Evidências de Validade e Precisão
}

\author{
Hugo Ferrari Cardoso ${ }^{1}$ \\ Universidade Estadual Paulista "Júlio de Mesquita Filho" - UNESP, Bauru-SP, Brasil \\ Makilim Nunes Baptista \\ Universidade São Francisco, Campinas-SP, Brasil
}

\section{RESUMO}

O artigo apresenta estudos de qualidades psicométricas para a Escala de Autoeficácia no Trabalho (EAE-T), sendo um de validade com base na estrutura interna (Análise Fatorial Exploratória- AFE e Análise Fatorial Confirmatória- AFC) e o outro de precisão por meio de consistência interna. No estudo de validade com base na AFE e precisão, a EAE-T foi aplicada em 525 trabalhadores e, como resultados, foi reduzida a 23 itens, distribuídos em dois fatores (fator 1, Execução no Trabalho, com cargas fatoriais variando entre 0,47 e 0,89, e o fator 2, Relações Interpessoais Trabalhistas, com cargas entre 0,56 e 0,85). Os coeficientes de precisão Orion foram de 0,96 (fator 1) e 0,95 (fator 2). Já na análise AFC, a EAE-T com 23 itens foi aplicada em 200 trabalhadores e verificou-se que todos os indicadores utilizados se mostraram com valores adequados, sendo que a estrutura (dois fatores) da EAE-T também foi confirmada. Palavras-chave: autoeficácia no trabalho; psicologia organizacional e do trabalho; avaliação psicológica.

\section{ABSTRACT - Work Self-Efficacy Scale: Validity and Accuracy Evidence}

The article presents psychometric quality studies for the Work Self-Efficacy Scale (WSES): one is a validity study based on the internal structure (Exploratory Factor Analysis, EFA, and Confirmatory Factor Analysis, CFA), and the other an accuracy study through internal consistency. In the validity study based on the EFA and accuracy, the WSES was applied in a sample of 525 workers, resulting in a reduction to 23 items, distributed in two factors (factor 1, Work Execution, with factorial loads varying between 0,47 and 0.89 , and factor 2, Interpersonal Labor Relations, with loads between 0.56 and 0.85 ). The Orion accuracy coefficients were 0.96 (factor 1 ) and 0.95 (factor 2). In the CFA, the WSES with 23 items was applied to a sample of 200 workers, and it was verified that all the indicators presented adequate values, and the structure (two factors) of the WSES was also confirmed.

Keywords: work self-efficacy; organizational and work psychology; psychological evaluation.

\section{RESUMEN - Escala de Autoeficácia no Trabalho: Evidencia de Validez y Precisión}

El artículo presenta estudios de calidad psicométrica en la Escala de Autoeficacia en el Trabajo (EAE-T), siendo uno de ellos, de validez con base en la estructura interna (Análisis Factorial Exploratorio - AFE y Análisis Factorial Confirmatorio - AFC) y el otro de precisión por medio de consistencia interna. En el estudio de validez basado en la AFE y precisión, la EAE-T fue aplicada en 525 trabajadores, y como resultado, se redujo a 23 ítems, distribuídos en dos factores (factor 1, Ejecución en el Trabajo, con cargas factoriales variando entre 0,47 y 0,89 , y factor 2 , Relaciones Interpersonales Laborales, con cargas entre 0,56 y 0,85 ). Los coeficientes de precisión Orion fueron de 0,96 (factor 1) y 0,95 (factor 2). En el análisis AFC, la EAE-T con 23 ítems fue aplicada en 200 trabajadores, y se verificó que todos los indicadores utilizados se mostraron con valores adecuados, y la estructura (dos factores) de la EAE-T también fue confirmada. Palabras clave: autoeficacia en el trabajo; psicología organizacional y del trabajo; evaluación psicológica.

Por autoeficácia entende-se, de acordo com autores expoentes na temática (Bandura, 1986, 1994, 1997, 2001; Pajares, 1997, 2002), como sendo crenças percebidas pelos indivíduos acerca de suas capacidades quanto a planejar e executar determinados comportamentos, visando atingir os objetivos almejados. Ao ser estudada no contexto organizacional, embora caiba aqui destacar que tal construto ainda não possui robusta literatura nesse meio, a autoeficácia tende a se apresentar como importante variável para os trabalhadores, associando-se positivamente com indicadores de engajamento no trabalho (Halbesleben, 2010; Sweetman \& Luthans, 2010; Xanthopoulou, Backker, Demerouti, \& Schaufeli, 2007), com a percepção de bem-estar e satisfação no trabalho (Erwins, 2001; Vinokur \& Schul, 2002) e com motivação no contexto laboral (LeBlanc, Schaufeli, Salanova, Llorens, \& Nap, 2010; Munir \& Nielsen, 2009; Salanova, Lorrente, Chambel, \& Matinezi, 2011). 
Embora seja percebida a importância desse construto, no Brasil, ao menos na literatura consultada para a realização deste estudo, não foram encontrados instrumentos que avaliassem a autoeficácia no trabalho. No contexto internacional, tomando como referência o trabalho de Cardoso, Baptista e Rueda (manuscrito não submetido), foram encontradas oito escalas. Tal manuscrito buscou analisar a literatura sobre o tema autoeficácia no trabalho, por meio de artigos publicados entre os anos de 2004 e 2014, indexados na base de dados EBSCO Academic Search, fazendo uso de duas palavras-chave para a busca ("self-efficacy" e "work"). O detalhamento acerca dos oito instrumentos de autoeficácia no trabalho encontrados na referida revisão pode visualizado na Tabela 1.

Tabela 1

Informações sobre Escalas de Autoeficácia no Trabalho Recuperadas da Literatura Internacional

\begin{tabular}{|c|c|c|c|c|}
\hline Referências & Instrumento & $\mathrm{NI}$ & Dimensões & Público avaliado \\
\hline 1 & $\begin{array}{l}\text { Return to Work } \\
\text { Self-Efficacy (RTW-SE) }\end{array}$ & 11 & Unidimensional & $\begin{array}{l}\text { Trabalhadores que } \\
\text { estejam voltando ao } \\
\text { trabalho após um } \\
\text { período de afastamento }\end{array}$ \\
\hline 2 & $\begin{array}{l}\text { Contextual Work Behavior } \\
\text { Self-Efficacy Scale (CWBSES) }\end{array}$ & 26 & $\begin{array}{l}4 \text { (Ajuste ocupacional; Ajuste } \\
\text { de performance; Adaptação } \\
\text { ao estresse; e Comparecimento) }\end{array}$ & $\begin{array}{l}\text { Trabalhadores } \\
\text { de forma geral }\end{array}$ \\
\hline 3 & $\begin{array}{l}\text { Work Self-Efficacy } \\
\text { Scale (WSES) }\end{array}$ & 10 & $\begin{array}{l}2 \text { (Relacionamento positivo e } \\
\text { Comprometimento) }\end{array}$ & $\begin{array}{l}\text { Trabalhadores } \\
\text { de forma geral }\end{array}$ \\
\hline 4 & $\begin{array}{l}\text { Self-Efficacy to } \\
\text { Manage Work-Family } \\
\text { Conflict (SE-WFC) }\end{array}$ & 10 & $\begin{array}{l}2 \text { (Autoeficácia- conflitos advindos } \\
\text { do trabalho e que podem interferir } \\
\text { no relacionamento familiar; } \\
\text { Autoeficácia- conflitos advindos } \\
\text { do relacionamento familiar } \\
\text { e que podem interferir na } \\
\text { execução das tarefas no trabalho) }\end{array}$ & $\begin{array}{l}\text { Trabalhadores } \\
\text { de forma geral }\end{array}$ \\
\hline 5 & $\begin{array}{l}\text { Community } \\
\text { Self-Efficacy } \\
\text { Scale (CSES) }\end{array}$ & 10 & Unidimensional & $\begin{array}{l}\text { Trabalhadores } \\
\text { voluntários }\end{array}$ \\
\hline 6 & $\begin{array}{l}\text { Norwegian Principal } \\
\text { Self-Efficacy Scale } \\
\text { (NPSES) }\end{array}$ & 22 & $\begin{array}{l}8 \text { (Gestão econômica; Liderança } \\
\text { instrucional; Autoridade } \\
\text { municipal; Relacionamento com } \\
\text { os pais; Comunidade local; Gestão } \\
\text { administrativa; Apoio ao } \\
\text { professor; Ambiente escolar) }\end{array}$ & $\begin{array}{l}\text { Diretores } \\
\text { de escolas }\end{array}$ \\
\hline 7 & $\begin{array}{l}\text { Work-Related } \\
\text { Self-Efficacy Scale } \\
\text { (WRSES) }\end{array}$ & 56 & $\begin{array}{l}6 \text { (Comunicação com clientes; } \\
\text { Avaliação das habilidades, } \\
\text { necessidades e outros recursos dos } \\
\text { clientes; Planejamento do processo } \\
\text { de intervenção; Realização da } \\
\text { intervenção; Aconselhamento, gestão } \\
\text { e supervisão; e Elaboração } \\
\text { de relatórios e documentação) }\end{array}$ & $\begin{array}{l}\text { Agentes } \\
\text { comunitários } \\
\text { de saúde }\end{array}$ \\
\hline 8 & $\begin{array}{l}\text { Occupational } \\
\text { Self-Efficacy Scale } \\
\text { (OSES) }\end{array}$ & 19 & $\begin{array}{l}6 \text { (Confiança; Comando/controle } \\
\text { sobre a situação; Capacidade de } \\
\text { adaptação; Eficácia pessoal; } \\
\text { Atitude positiva; e Individualidade) }\end{array}$ & $\begin{array}{l}\text { Trabalhadores } \\
\text { de forma geral }\end{array}$ \\
\hline
\end{tabular}

Referências. 1 (Besen, Young, \& Shaw, 2015; Huijs, Koppes, Taris, \& Blonk, 2012; Lagervelda, Blonka, Brenninkmeijerb, \& Schaufelib, 2010; Shaw, Reme, Linton, \& Pransky, 2011); 2 (O’Sullivan, Strauser, \& Wong, 2012; Strauser, O’Sullivan, \& Wong, 2010); 3 (Pepe, Farnese, Avalone, \& Vecchione, 2010); 4 (Colombo \& Zito, 2014; Hennessy \& Lent, 2008; Rauf, 2013); 5 (Bai \& Stewart, 2010; DePrince, Priebe, \& Newton, 2011; Ferrari, 2004; Piñgul, 2015; Reeb, Folger, Langsner, Ryan, \& Crouse, 2010); 6 (Federici \& Skaalvik, 2011, 2012 ); 7 (Vax, Schreuer, \& Sachs, 2012; Waghorn, Chant, \& King, 2005); 8 (Chaudhary, Rangnekar, \& Barua, 2012); NI: Número de itens do instrumento

Acerca das informações disponibilizadas na Tabela 1, algumas constatações podem ser observadas. A primeira delas diz respeito ao número de itens dos instrumentos.
Das oito escalas apresentadas, três (maior frequência) são compostas por 10 itens, além disso, apenas a WRSES apresenta um número bastante superior de itens (56 no 
total), o restante possui uma variação entre 11 e 26 itens. Em outras palavras, os instrumentos que avaliam autoeficácia aqui visualizados não apresentam um número elevado de itens. A segunda constatação foi que, em geral, os instrumentos de autoeficácia no trabalho possuem o formato de respostas do tipo Likert com variações entre 5 e 10 pontos.

Uma terceira constatação foi que, em sua maioria, as escalas apresentam mais de uma dimensão (apenas duas são unifatoriais, o restante teve uma variação entre duas e oito dimensões). Percebe-se também que não há necessariamente semelhanças, com base nas nomenclaturas dos fatores, em relação ao que é avaliado por intermédio dessas dimensões. Ou seja, embora sejam escalas que se propõem avaliar a autoeficácia no trabalho, elas mensuram aspectos diversos da temática, não se assemelhando em alguns pontos.

Em outras palavras, embora a autoeficácia seja vista conceitualmente como a percepção acerca da capacidade em planejar e executar comportamentos visando atingir os objetivos almejados (Bandura, 1986), alguns instrumentos avaliam tais comportamentos em uma única dimensão, outras os investigam agrupando-os em situações específicas, muitas dessas relacionadas às esferas do contexto de trabalho. A seguir, com o intuito de mostrar ao leitor os principais fatores investigados em autoeficácia no trabalho, eles serão elucidados.

Em uma tentativa de agrupar as dimensões de todos os instrumentos em categoriais mais amplas, com base na Tabela 1, pode-se verificar a avaliação de (a) aspectos relacionados à capacidade de execução das atividades laborais (dimensões Ajuste ocupacional e Ajuste de performance - da CWBSES; comprometimento - dimensão da WSES; Avaliação das habilidades, necessidades e outros recursos dos clientes, Planejamento do processo de intervenção; Realização da intervenção e Elaboração de relatórios e documentação - fatores da WRSES; e Capacidade de adaptação - OSES); (b) resiliência para se adaptar às situações estressoras e conflituosas (dimensão Adaptação ao estresse da CWBSES e as duas dimensões da SE-WFC); (c) características pessoais que podem resultar em maior eficácia no trabalho (dimensão Comparecimento - CWBSES; fatores Confiança, Eficácia pessoal, Atitude positiva e Individualidade OSES); (d) questões ligadas à capacidade de gestão no trabalho (dimensões Gestão econômica, Liderança instrucional, Autoridade municipal, Gestão administrativa da NPSES; fator Aconselhamento, gestão e supervisão - WRSES; e Comando/controle sobre a situação, avaliada pela OSES);(e) aspectos relacionados às interações sociais no trabalho (dimensão Relacionamento positivo da WSES; Relacionamento com os pais e Apoio ao professor - NPSES); (f) eficácia na comunicação (fator Comunicação com clientes - WRSES); além de (g) contextos específicos (como Comunidade local e Ambiente escolar, ambas avaliadas pela NPSES).
Quanto ao público para o qual os instrumentos são direcionados, constatou-se que a maior frequência não restringe a um único grupo de trabalhadores (na Tabela 1 foi nomeado como trabalhadores de forma geral). Entretanto, algumas escalas se propõem avaliar públicos bem direcionados, como voluntários e agentes comunitários de saúde, por exemplo.

Em síntese, é possível afirmar que, embora a temática autoeficácia no trabalho seja importante, uma vez que pode contribuir, além do engajamento e motivação, também para a percepção de saúde e bem-estar dos trabalhadores, até o presente momento, ainda se encontra pouco estudada por investigações científicas (tanto no Brasil, como no contexto internacional). Em especial, poucos são os instrumentos construídos e com propriedades psicométricas comprovadas para a mensuração de tal construto.

Com base nessas conclusões, Cardoso e Baptista (2014) iniciaram a construção de um instrumento cujo objetivo é a avaliação da autoeficácia no trabalho, sem que houvesse a determinação de um público em específico (trabalhadores de forma geral). Tal instrumento foi nomeado inicialmente como Escala de Autoeficácia no Trabalho (EAE-T). As justificativas para a condução da construção da escala se sustentam na medida em que não há registros de instrumentos brasileiros que avaliem autoeficácia no trabalho (existem escalas que avaliam autoeficácia geral, ou mesmo para se buscar um emprego e fazer uma escolha profissional, mas não uma específica ao contexto de trabalho).

Além disso, os autores da EAE-T chegaram à conclusão que a adaptação de escalas internacionais poderia levar em consideração algumas dimensões que não necessariamente se mostram condizentes às estudadas no Brasil, na área organizacional e do trabalho. De forma mais específica, para a construção da EAE-T, tomou-se como base a teoria de autoeficácia de Bandura (1986), assim como foram investigados os instrumentos descritos na Tabela 1 e diversos instrumentos da área de Psicologia Organizacional e do Trabalho (POT) construídos no Brasil (a fim de se identificar as principais dimensões que pudessem estar relacionadas ao conceito de autoeficácia no trabalho).

Os instrumentos da área de POT que foram analisados e consultados em duas obras têm como propósito a apresentação de diversas escalas construídas no Brasil (Siqueira, 2008a; 2014). Foi realizado tal levantamento dos instrumentos nacionais, visando verificar as principais dimensões mensuradas por tais escalas, ou seja, uma vez que a EAE-T é uma escala construída no Brasil, e direcionada para o contexto organizacional e do trabalho, tornou-se importante se averiguar quais fatores avaliados pelas escalas brasileiras que poderiam estar relacionados com a autoeficácia no trabalho. No total, foram avaliados 10 instrumentos e suas respectivas dimensões, aos quais avaliam clima organizacional (Martins, 2008), comprometimento organizacional (Bastos, Siqueira, Medeiros, \& Menezes, 2008), satisfação no trabalho (Siqueira 2008b), 
contexto do trabalho (Ferreira \& Mendes, 2008), cultura organizacional (Ferreira \& Assmar, 2008), bem-estar no trabalho (Siqueira, Orengo, \& Pieró, 2014), cidadania organizacional (Bastos, Siqueira, \& Gomes, 2014), engajamento no trabalho (Siqueira, Martins, Orengo, \& Souza, 2014), políticas e práticas de recursos humanos (Demo, Neiva, Nunes, \& Rozzett, 2014) e socialização organizacional (Borges, Silva, Melo, \& Martins, 2014).

Após a análise dos instrumentos do contexto organizacional, bem como as escalas internacionais que avaliam autoeficácia no trabalho (apresentadas na Tabela 1), os quais serviram como parâmetro para se refletir acerca de possíveis agrupamentos de itens, deu-se o início da construção da EAE-T fazendo uso para a elaboração do itens dos pressupostos de Bandura (1986), cujo objetivo foi verificar a capacidade percebida pelo trabalhador em diferentes situações do seu contexto laboral, tendo como base cinco possíveis agrupamentos, quais sejam, 1. fatores relacionados às tarefas de trabalho; 2 . fatores relacionados às normas da organização; 3 . políticas e práticas de gestão de pessoas; 4 . fatores relacionados às relações interpessoais no trabalho; e 5. relacionamento com a chefia. Assim, a proposta do presente estudo consiste em apresentar estudos de evidência de validade com base na estrutura interna, por meio de análises fatoriaisexploratória e confirmatória, além de estudo de precisão, utilizando-se o procedimento de consistência interna.

\section{Etapa 1. Análise Fatorial Exploratória da EAE-T}

\section{Método}

\section{Participantes}

Participaram da pesquisa 525 trabalhadores com idades variando entre 18 e 66 anos $(M=33,23 ; D P=10,56)$, e escolaridade desde o ensino fundamental incompleto até o ensino superior completo, sendo a maior parte $(n=364$, representando 69,3\%) de trabalhadores com ensino superior completo. Quanto ao sexo, a maior parte, $304(57,9 \%)$ era mulheres. No que se refere ao estado civil dos participantes, as maiores frequências foram de solteiros, com 272 (51,8\%), seguidos de casados, 208 $(39,6 \%)$. Das variáveis organizacionais, no que tange ao tipo de organização, a maior parte era de empresas privadas $(n=339 ; 64,6 \%)$, seguida por empresas públicas $(n=150 ; 28,6 \%)$ e terceiro setor $(n=35 ; 6,7 \%)$. Do ramo de atuação de tais organizações, houve predominância de empresas prestadoras de serviços $(n=367 ; 69,9 \%)$.

\section{Instrumento}

Escala de Autoeficácia no Trabalho - EAE-T (Cardoso \& Baptista, 2014). A EAE-T foi construída considerando a teoria de autoeficácia proposta por Bandura (1986). Para tanto, os itens foram elaborados tendo como base cinco possíveis agrupamentos, quais sejam, 1 . fatores relacionados às tarefas de trabalho; 2 . fatores relacionados às normas da organização; 3 . políticas e práticas de gestão de pessoas; 4. fatores relacionados às relações interpessoais no trabalho; e 5. relacionamento com a chefia. A versão aplicada na Etapa 1, primeira versão da EAE-T, ficou composta por 42 itens, respondidos por meio de opções respostas Likert, sendo estas, nada; pouco; mais ou menos; muito; extremamente. Em cada item, o trabalhador deveria responder, com base nas opções apresentadas, o quanto ele se percebe capaz de executar.

\section{Procedimentos}

Após a aprovação do projeto pelo comitê de ética de pesquisa (protocolo número 45431815.0.0000.5398), os dados foram coletados em organizações de diferentes ramos de atuação. Foi aplicado um protocolo contendo o Termo de Consentimento Livre e Esclarecido - TCLE (como condicionante para a participação) e a Escala de Autoeficácia no Trabalho (EAE-T). Os dados foram coletados de forma individual e coletiva (não ultrapassando o número de 30 pessoas no caso da aplicação coletiva), dependendo da disponibilidade oferecida por cada organização, sendo que, em média, o tempo de aplicação foi de 20 minutos. Cabe destacar que, em ambos os casos de aplicação, individual e coletiva, os pesquisadores explicaram os objetivos da pesquisa, solucionaram eventuais dúvidas dos participantes, e a coleta só foi iniciada após a concordância do mesmo (por meio do TCLE).

\section{Análise de Dados}

Para estudar a estrutura interna da EAE-T, foi realizada uma análise fatorial exploratória (AFE), utilizando o método de extração Robust Unweighted Least Squares (RULS), com rotação promin e correlações policóricas, procedimentos adequados ao nível de medida ordinal. A análise da dimensionalidade também foi investigada por meio do procedimento de análise paralela, utilizando o programa Factor. Os fatores gerados da EAE-T foram analisados utilizando a correlação de Pearson. Por fim, quanto aos índices de precisão, eles foram calculados por meio do coeficiente Orion.

\section{Etapa 2. Análise Fatorial Confirmatória da EAE-T}

\section{Método}

\section{Participantes}

Uma nova coleta de dados foi realizada e, da etapa 2 , fizeram parte da amostra 200 trabalhadores com idades variando entre 18 e 63 anos $(M=33,34 ; D P=10,43)$, e escolaridade desde o ensino fundamental incompleto até a ensino superior completo, sendo a maior parte $(n=132$, representando $66,0 \%)$ de trabalhadores com ensino superior completo. Quanto ao sexo, 104 (52\%) eram homens e $96(48 \%)$ mulheres. No que se refere ao estado civil dos participantes, as maiores frequências foram de solteiros, com 94 (47\%), seguidos de casados, 
88 (44\%). Das variáveis organizacionais, no que tange ao tipo de organização, a maior parte era de empresas privadas $(n=122 ; 61 \%)$, seguida por empresas públicas $(n=54$; $27 \%)$ e terceiro setor $(n=24 ; 12 \%)$. Do ramo de atuação de tais organizações as maiores frequências foram de empresas prestadoras de serviços $(n=95 ; 47,5 \%)$, seguido de indústrias $(n=59 ; 29,5 \%)$ e comércio $(n=46 ; 23 \%)$.

\section{Instrumento}

Escala de Autoeficácia no Trabalho - EAE-T (Versão após AFE da Etapa 1). A EAE-T aplicada na etapa 2 era composta por 23 itens, distribuídos em dois fatores (versão após AFE - etapa 1). O primeiro fator da EAE-T ficou composta por 13 itens que dizem respeito a execução do trabalho. O segundo fator ficou composta por 10 itens relacionados a relações interpessoais trabalhistas. Todos os itens são respondidos por intermédio de uma escala Likert (os mesmos da escala aplicada na etapa 1 deste artigo).

\section{Procedimentos}

A coleta dos dados para a segunda etapa da pesquisa se deu de forma semelhante aos procedimentos adotados na etapa 1 deste artigo. Os dados foram coletados em organizações de diferentes ramos de atuação. Foi aplicado um protocolo contendo o Termo de Consentimento Livre e Esclarecido - TCLE (como condicionante para a participação) e a EAE-T (versão após AFE - com 23 itens). Os dados foram coletados de forma individual e coletiva, dependendo da disponibilidade oferecida por cada organização, sendo que, no caso da aplicação coletiva, o número não foi superior a 30 pessoas. Em média o tempo de aplicação foi de dez minutos.

\section{Análise dos Dados}

Para proceder a AFC, foi utilizado o programa estatístico Mplus versão 7.11. Testou-se, por intermédio do programa do estimador Weighted Least Squares Meanand Variance Adjusted (WLSMV) a estrutura de dois fatores para os itens que compõem a EAE-T. De forma adicional, para a verificação da consistência dos dados aglutinados em duas dimensões, utilizou-se os índices de qui-quadrado norma do $\left(\chi^{2} / g l\right)$, o índice de ajuste comparativo (CFI), a raiz da média dos quadrados dos erros de aproximação (RMSEA, com intervalo de confiança de 95\%) e o índice Tucker Lewis (TLI).

\section{Etapa 1. Resultados da AFE da EAE-T}

\section{Resultados}

Primeiramente, buscou-se verificar a possibilidade de fatorabilidade dos itens da EAE-T. Assim, procedeu-se as análises por meio do Kaiser-Meyer-Olkin (KMO) e a prova de esfericidade de Bartlett. Dos resultados, o KMO foi igual a 0,94 , enquanto que a prova de esfericidade de Bartlett foi estatisticamente significativa $\chi^{2}=5091,5$ $(d f=253 ; p<0,001)$. Com base no exposto, ambos os critérios sustentaram a fatorabilidade da escala.

Posteriormente, procedeu-se a AFE solicitando-se a retenção de itens cujas cargas fatoriais fossem superiores a 0,45 . Esse procedimento resultou na retirada de 16 itens, restando 26 , que foram analisados de forma a identificar o número de fatores que comporiam a escala. Para definir o número de fatores, foram utilizados dois critérios, quais sejam, o de Guttman-Kaiser e a análise paralela. Considerando o critério de Guttman-Kaiser, ou da raiz latente, foram identificados sete fatores com autovalores acima de 1,0. Entretanto, seguindo recomendações de Damásio (2012), optou-se por considerar os dados da análise paralela, pois o critério de Kaiser-Guttman tende a superestimar o número de fatores a ser retido devido ao erro amostral.

Pela análise paralela, procedeu-se os métodos de Hull (Lorenzo-Seva, Timmerman, \& Kiers, 2011), Minimum Average Partial - MAP (Velicer, 1976) e Optimal Implementation of Parallel Analysis - PA (Timmerman \& Lorenzo-Seva, 2011) para se verificar a dimensionalidade da escala. Dos resultados, os métodos de Hull e PA indicaram pela unidimensionalidade da EAE-T, enquanto o MAP indicou a existência de dois fatores no instrumento. Embora em dois métodos observou-se a unidimensionalidade, assumiu-se dois fatores para a EAE-T pelo fato deles apresentarem sustentação teórica. É pertinente esclarecer que a sustentação teórica para a escolha de dois fatores foi baseada nos critérios de MAP.

Considerando a estrutura em dois fatores, dos 26 itens analisados, três foram retirados (por estarem configurados em mais de um fator ou pelo fato de não se agrupar a nenhuma dimensão). Os 23 itens restantes da EAE-T podem ser observados na Tabela 2.

É possível verificar, na Tabela 2, a existência de dois fatores da EAE-T, nos quais estão aglutinados os 23 itens da escala. O primeiro fator, denominado "execução do trabalho", ficou composto por 13 itens, tendo suas cargas fatoriais variado entre 0,46 e 0,88 , autovalor de 10,79 e $46,91 \%$ da variância explicada. Essa dimensão visa avaliar o quanto o trabalhador se percebe capaz de desenvolver de forma adequada suas atividades no trabalho. Como exemplos de itens, "Pensar em alternativas para resolver problemas da melhor forma possível em meu trabalho", "Executar o que deve ser feito em meu trabalho" e "Me organizar para que meu trabalho não fique atrasado".

A segunda dimensão, relações interpessoais trabalhistas, ficou composta por 10 itens, tendo suas cargas fatoriais variado entre 0,56 e 0,84 , autovalor de 1,79 e $7,81 \%$ da variância explicada. Tal dimensão diz respeito ao quanto o trabalhador se percebe capaz de se relacionar de forma eficaz com os demais colegas que convive no ambiente laboral. Como exemplos de itens, pode-se citar "Me comunicar adequadamente com meus colegas de trabalho", "Trabalhar em equipe" e "Trabalhar com pessoas mais velhas do que eu". 
Tabela 2

Cargas Fatoriais por Meio da AFE, com Rotação Promin, e Valores das Comunalidades

\begin{tabular}{|c|c|c|c|}
\hline Componentes & 1 & 2 & $\mathrm{~h}^{2}$ \\
\hline Item 11 & 0,88 & & 0,80 \\
\hline Item 10 & 0,85 & & 0,80 \\
\hline Item 22 & 0,78 & & 0,81 \\
\hline Item 19 & 0,68 & & 0,68 \\
\hline Item 4 & 0,68 & & 0,70 \\
\hline Item 21 & 0,69 & & 0,82 \\
\hline Item 9 & 0,61 & & 0,62 \\
\hline Item 3 & 0,62 & & 0,75 \\
\hline Item 23 & 0,63 & & 0,86 \\
\hline Item 12 & 0,62 & & 0,88 \\
\hline Item 15 & 0,57 & & 0,79 \\
\hline Item 16 & 0,59 & & 0,58 \\
\hline Item 20 & 0,46 & & 0,62 \\
\hline Item 6 & & 0,84 & 0,87 \\
\hline Item 1 & & 0,75 & 0,85 \\
\hline Item 18 & & 0,78 & 0,79 \\
\hline Item 5 & & 0,70 & 0,63 \\
\hline Item 8 & & 0,74 & 0,79 \\
\hline Item 2 & & 0,78 & 0,88 \\
\hline Item 13 & & 0,63 & 0,81 \\
\hline Item 17 & & 0,71 & 0,86 \\
\hline Item 14 & & 0,62 & 0,73 \\
\hline Item 7 & & 0,56 & 0,85 \\
\hline Quantidade de itens & 13 & 10 & \\
\hline Eigenvalue & 10,79 & 1,79 & \\
\hline Variância explicada (\%) & 46,91 & 7,81 & \\
\hline Total de Variância explicada (\%) & \multicolumn{2}{|c|}{54,72} & \\
\hline
\end{tabular}

Os dois agrupamentos de itens (execução do trabalho e relações interpessoais trabalhistas) apresentaram coeficiente de correlação de Pearson de 0,77, com significância estatística $(p<0,001)$. De acordo com Dancey e Reidy (2006), correlações acima de 0,70 são consideradas de magnitudes altas. Dessa forma, as duas dimensões da EAE-T se propõem a verificarem percepção de autoeficácia no trabalho, ou seja, as dimensões advindas das análises fatoriais mensuram a capacidade percebida pelos indivíduos em situações específicas em seu contexto laboral. A primeira está relacionada ao quanto trabalhador se percebe capaz em desempenhar suas funções, na execução de suas atividades de trabalho, e a segunda dimensão mensura a capacidade percebida por ele em interagir positivamente com os demais colegas de trabalho e lideranças.

Quanto ao procedimento de verificação do coeficiente de precisão para a EAE-T, por meio da verificação do índice de precisão por Orion, os itens da escala foram analisados em seus agrupamentos (dimensões). A primeira, execução no trabalho, composto por 13 itens, obteve índice de fidedignidade de 0,96 . a segunda dimensão, relações interpessoais trabalhistas, contém 10 itens e 0,95. Já a confiabilidade ômega foi de 0,95 para a EAE-T, escala em sua totalidade

\section{Etapa 2. Resultados da AFC da EAE-T}

A análise fatorial confirmatória foi realizada por intermédio do programa Mplus versão 7.11 (Muthén \& Muthén, 2012), sendo utilizado como estimador o Weighted Least Squares Meanand Variance Adjusted (WLSMV). Foram verificadas algumas medidas para se constatar a qualidade de ajuste entre o modelo proposto e os dados obtidos por meio da amostra, sendo esses o qui-quadrado norma do - qui quadrado sobre graus de liberdade $\left(\chi^{2} / g l\right)$, o índice de ajuste comparativo (CFI), a raiz da média dos quadrados dos erros de aproximação (RMSEA) e o índice Tucker Lewis (TLI).

$\mathrm{O} \chi^{2} / g l$ divide o valor do qui-quadrado pelo número de graus de liberdade para obter um valor de ajuste ao modelo menos sensível ao tamanho da amostra, ou seja, torna-se o qui-quadrado menos dependente do tamanho amostral. De acordo com Byrne (2001), valores 
menores que 3,0 são preferíveis nesse tipo de análise. Já para Hair, Anderson, Thatam e Black (2005), o valor mínimo aceitável é 1,0, sendo que valores máximos variam entre 2 e 3. Na análise em relação aos dados da EAE-T, observou-se o valor de $\chi^{2} / g l$ de 2,30, considerado adequado em todos os parâmetros anteriormente apresentados.

O CFI é um índice de ajuste comparativo, por meio do qual se compara o ajuste do modelo existente a um modelo nulo, fazendo para tal o uso de uma distribuição de qui-quadrado não central, procurando levar em consideração a complexidade do modelo. O CFI, dessa forma, assume que os indicadores e também as variáveis latentes não são correlacionados. Para esse tipo de análise, Hu e Bentler (1999) e Thompson (2004) ressaltaram que valores a partir de 0,90 são desejados (sendo que iguais ou maiores que 0,95 apresentam maior consistência), uma vez que indicam um bom ajuste do modelo proposto. $\mathrm{Na}$ presente pesquisa, o índice CFI obtido foi de 0,92 , próximo ao índice ideal.

O terceiro indicador analisado na presente pesquisa, o RMSEA, diz respeito a raiz da média dos quadrados dos erros de aproximação, sendo um valor representativo da qualidade do ajuste que poderia ser esperado para os dados, caso o modelo fosse estimado na população.
De acordo com Hair et al. (2005) e Thompson (2004), o RMSEA é um índice que verifica a estimação dos dados para além da amostra investigada, mas também verifica o quão bem o modelo se ajusta à população de interesse. Valores iguais ou abaixo a 0,08 são considerados desejáveis para esses indicadores. $\mathrm{O}$ valor obtido por intermédio da análise de dados da EAE-T foi de 0,08, estando no limite do considerado adequado, segundo os autores.

Por fim, o índice TLI também é uma medida que compara o modelo proposto com um modelo nulo, assim como o CFI. No presente estudo, o TLI foi utilizado como medida de comparação entre os modelos de um e de dois fatores. Segundo Bentler e Bonett (1980) e Hair et al. (2005), para análise de TLI são recomendados valores superiores a 0,90 . Dos dados de TLI, o modelo com dois fatores apresentou um índice mais adequado, com TLI de 0,92. A seguir, a Tabela 3 apresentará a distribuição dos itens da EAE-T após procedimento de análise fatorial confirmatória.

A partir dos resultados obtidos na AFC, pode-se destacar que a estrutura bifatorial da EAE-T foi confirmada de forma consistente. Como pode ser visto, a mesma estrutura fatorial (bifatorial) foi constatada tanto na AFE como na AFC.

Tabela 3

Distribuição dos Itens da EAE-T após AFC

\begin{tabular}{|c|c|c|}
\hline Componentes & 1 & 2 \\
\hline Item 11 & 0,60 & \\
\hline Item 10 & 0,62 & \\
\hline Item 22 & 0,63 & \\
\hline Item 19 & 0,66 & \\
\hline Item 4 & 0,67 & \\
\hline Item 21 & 0,68 & \\
\hline Item 9 & 0,70 & \\
\hline Item 3 & 0,72 & \\
\hline Item 23 & 0,72 & \\
\hline Item 12 & 0,73 & \\
\hline Item 15 & 0,75 & \\
\hline Item 16 & 0,77 & \\
\hline Item 20 & 0,79 & \\
\hline Item 6 & & 0,60 \\
\hline Item 1 & & 0,65 \\
\hline Item 18 & & 0,67 \\
\hline Item 5 & & 0,68 \\
\hline Item 8 & & 0,69 \\
\hline Item 2 & & 0,73 \\
\hline Item 13 & & 0,74 \\
\hline Item 17 & & 0,75 \\
\hline Item 14 & & 0,77 \\
\hline Item 7 & & 0,79 \\
\hline Quantidade de itens & 13 & 10 \\
\hline
\end{tabular}




\section{Discussão}

O presente artigo apresentou as informações referentes a dois estudos de qualidades psicométricas para a EAE-T, sendo um de validade com base na estrutura interna (AFE e AFC) e o outro de precisão por meio de consistência interna. Compete destacar a pertinência para a construção desse instrumento na medida em que a autoeficácia no trabalho se mostra como sendo um construto relevante, uma vez que pode influenciar no desempenho, persistência e motivação das pessoas para a realização de determinadas tarefas, inclusive no trabalho (Bandura, Barbarabelli, Caprara, \& Pastorelli, 2001).

Para a realização da AFE, utilizou-se procedimentos de análise do índice Kaiser-Meyer-Olkin (KMO) e a prova de esfericidade de Bartlett, visando atestar a possibilidade de fatorabilidade dos itens. Os dois critérios foram considerados satisfatórios $(\mathrm{KMO}=0,94$, prova de esfericidade de Bartlett foi estatisticamente significativa - 5091,5; $p<0,001$ ), os quais sustentaram a possibilidade de fatorabilidade do conjunto de itens da EAE-T (Pereira, 1999). Posteriormente, foram analisados os itens e fatores, sendo que se adotou como critérios o fato de que os itens deveriam apresentar cargas fatoriais superiores a 0,45 e, para a definição de fatores, utilizou-se os critérios relativos à análise paralela e teste de Guttman-Kaiser, por meio dos quais resultou-se na EAE-T com sua estrutura em dois fatores e composta por 23 itens (Damásio, 2012).

Das duas dimensões da EAE-T, a primeira, denominada execução do trabalho, consiste em averiguar a percepção acerca da capacidade do indivíduo em desenvolver suas funções laborais. Esse fator também pode ser visualizado em outras escalas que mensuram a autoeficácia no trabalho e que foram apresentadas na Tabela 1, tais como a CWBSES (Strauser, O'Sullivan, \& Wong, 2010), com as dimensões Ajuste ocupacional e Ajuste de performance; a WSES (Avallone, Pepe, \& Porcelli, 2007) com o fator Comprometimento; a escala WRSES (Waghorn et al., 2005) com suas dimensões Avaliação das habilidades, necessidades e outros recursos dos clientes, Planejamento do processo de intervenção; Realização da intervenção e Elaboração de relatórios e documentação; e o instrumento OSES (Schyns \& von Collani, 2002) por meio da dimensão Capacidade de adaptação. Nota-se que a avaliação dos aspectos relacionados à execução das tarefas no trabalho é de grande importância na mensuração da autoeficácia no trabalho, uma vez que, das oito escalas apresentadas na Tabela 1, ao menos metade destas, apresentam itens direcionados para a avaliação de tais aspectos.

Já a segunda dimensão da EAE-T, relações interpessoais trabalhistas, tem como propósito a mensuração acerca da percepção de capacidade dos trabalhadores em se relacionar de forma eficaz com os demais colegas que convivem no ambiente laboral. De forma semelhante ao que foi visto na discussão da dimensão execução do trabalho, esse segundo fator da EAE-T também pode ser constatado em instrumentos de autoeficácia no trabalho já apresentados (Tabela 1). De forma mais específica, possuem itens relacionados às interações sociais no trabalho as escalas WSES (Avallone et al., 2007), com a dimensão relacionamento positivo, a NPSES (Federici \& Skaalvik, 2011), por meio dos fatores relacionamento com os pais e apoio ao professor.

Quanto à correlação entre os fatores, de acordo com Dancey e Reidy (2006), a magnitude foi moderada execução do trabalho e relações interpessoais trabalhistas. A esse respeito, pelo fato da autoeficácia no trabalho ser considerado um construto multidimensional, era realmente esperado que as correlações entre os fatores fossem de magnitudes classificadas como alta (uma vez que possuem consistentes associações). Conforme já destacado, a autoeficácia é conceitualmente caracterizada pela percepção acerca da capacidade em planejar e executar comportamentos, visando atingir aos objetivos almejados (Bandura, 1986), e como os itens foram construídos com base em tal conceito e adaptados ao contexto organizacional, era esperada correlação de magnitude alta (uma vez que ambas as dimensões se propõem avaliar a autoeficácia no trabalho).

Assim, o estudo de AFE resultou em uma escala de 23 itens, o que pode ser considerado como similar às características das demais escalas de autoeficácia no trabalho aqui apresentadas (com grande parte dos instrumentos variando 10 e 22 itens). A esse respeito, utilizando como base os instrumentos de autoeficácia no trabalho apresentados, percebe-se que em geral tais escalas tendem a ter poucos itens, ou seja, de rápida aplicação. Além disso, os itens aglutinados nas duas dimensões da EAE-T apresentaram consistência semântica em relação a cada fator, bem como há um equilíbrio entre número de itens nas dimensões, 13 (execução do trabalho) e 10 (relações interpessoais trabalhistas).

O estudo de precisão foi atestado por meio da consistência interna, mais especificamente, fazendo uso do procedimento de averiguação por meio dos valores Orion. Segundo Pasquali (1999), para esse tipo de investigação, são aceitáveis índices de precisão acima de 0,70. Esse mesmo parâmetro também é reconhecido pelo Conselho Federal de Psicologia (CFP) por meio do Sistema de Avaliação de Testes Psicológicos (SATEPSI, 2004). Como pode ser visualizado nos resultados deste estudo, a EAE-T apresentou índices de precisão superiores a $0,70 \mathrm{em}$ seus dois fatores, atestando, dessa forma, condições adequadas de precisão via consistência interna.

O segundo estudo de análise fatorial (confirmatória) da EAE-T também obteve resultados satisfatórios. Algumas discussões já foram feitas no tópico de resultados, porém alguns pontos serão brevemente retomados neste tópico. Todos os índices investigados na AFC 
estiveram dentro dos parâmetros considerados adequados, a saber, para o $\chi^{2} / g l$ de acordo com os autores (Byrne, 2001; Hair et al., 2005) espera-se valores menores que três, sendo que a EAE-T obteve 2,30; para o CFI é esperado um valor superior 0,90 , sendo desejável um valor acima de 0,95 (Hu \& Bentler, 1999; Thompson, 2004) e, nesse caso, a EAE-T obteve um valor $(0,92)$ próximo a esse índice; quanto à RMSEA, que era esperado um valor igual ou inferior a 0,08 (desejável segundo Hair et al., 2005 e Thompson, 2004), a EAE-T obteve o índice de 0,08 ; já no TLI, em que era esperado valores superiores a 0,90, desejáveis de acordo com os autores (Bentler \& Bonett, 1980; Hair et al., 2005), a EAE-T obteve nesse parâmetro o valor 0,92 .

Todas essas informações da AFC confirmam o modelo estimado em dois fatores, sendo esses os mesmos obtidos pela AFE. Assim, pode-se constatar que a presente pesquisa cumpriu com os objetivos de verificar a evidência de validade com base na estrutura interna (fazendo uso de dois procedimentos para tal - AFE e AFC), além de também obter índices considerados satisfatórios e assim atestar a precisão com base na consistência interna (fazendo uso da verificação do índice Orion). Por fim, este estudo, apesar de trazer uma estrutura fatorial exploratória relativamente estável, indicada também pela análise confirmatória, não pode caracterizar o construto a partir dessa estrutura encontrada. Como observado anteriormente, estruturas diversas podem ser encontradas em outras escalas de autoeficácia no trabalho. Provavelmente, os diversos resultados fatoriais podem ser explicados por diferentes teorias de base, amostras e talvez características das culturas e comportamentos organizacionais.

\section{Considerações Finais}

A pesquisa em questão objetivou apresentar dois estudos de qualidades psicométricas, sendo esses a análise com base na estrutura interna (fazendo uso de análises fatoriais - exploratória e confirmatória) e o estudo de fidedignidade por meio do índice Orion. Percebeu-se que os itens da EAE-T se agruparam de forma coerente às duas dimensões geradas pelas análises fatoriais, assim como os índices de precisão dos fatores se mostraram acima dos parâmetros considerados mínimos na literatura.

Embora os objetivos do presente estudo tenham sido contemplados, alguns tópicos podem ser sinalizados como forma de se atentar para estudos futuros. Assim, se fazem necessários novos estudos, visando principalmente verificar as evidências de validade com base relação com outras variáveis para a EAE-T, podendo essa ser associada a outros instrumentos de autoeficácia geral, bem como variáveis organizacionais, como motivação e engajamento no trabalho. Além disso, torna-se imprescindível realizar investigações com um número amostral maior, e em diferentes regiões do país, com maior variabilidade da variável escolaridade (uma vez que, nas amostras da presente pesquisa, a maior parte dos participantes tinha ensino superior completo), em diferentes áreas de atuação das organizações (tais como comércio, indústria e prestação de serviços) com o intuito de buscar a normatização da EAE-T.

\section{Referências}

Avallone, F., Pepe, S., \& Porcelli, R. (2007). Autoefficacia per cepitan ellaricerca del lavoro: Scale di misura. EmIsfol, Bisogni, valori e autoefficacian ellascelta del lavoro. Roma: ISFOL, 133-142. Recuperado de http://hdl.handle.net/11573/364169.

Bai, H., \& Stewart, T. (2010). A psychometric study of the community service self-efficacy scale for evaluation of service-learning programs. Citizenship, Social and Economics Education, 9(2), 115-128.doi: 10.2304/csee.2010.9.2.115

Bandura, A. (1986). Social foundations of thought and action: A social cognitive theory. Englewood Cliffs, NJ: Prentice-Hall.

Bandura, A. (1994). Self-Efficacy. Em V. S. Ramachaudran (Ed.), Encyclopeida of human behavior, (Vol. 4, pp. 71-81). San Diego: Academic Press.

Bandura, A. (1997). Self-efficacy: The exercise of control. New York: Freeman.

Bandura, A. (2001) Social cognitive theory: An agentic perspective. Annual Review of Psychology, 52, 1-26. doi: 10.1146/annurev.psych.52.1.1

Bandura, A., Barbarabelli, C., Caprara, G. V., \& Pastorelli, C. (2001). Self-efficacy beliefs as shapers of children_s aspirations and career trajectories. Child Development, 72(1), 187-206. doi: 10.1111/1467-8624.00273.

Bastos, A. V. B., Siqueira, M. M. M., \& Gomes, A. C. P. (2014). Cidadania organizacional. Em Siqueira, M. M. M. (Ed.), Novas medidas do comportamento organizacional: ferramentas de diagnóstico e gestão (pp. 79-103). Porto Alegre: Artmed.

Bastos, A. V. B., Siqueira, M. M. M., Medeiros, C. A. F., \& Menezes, I. G. (2008). Comprometimento organizacional. Em Siqueira, M. M. M. (Eds.), Medidas do comportamento organizacional: ferramentas de diagnóstico e gestão (pp. 49-96). Porto Alegre: Artmed.

Bentler, P. M., \& Bonett, D. G. (1980). Significance tests and goodness of fit in the analysis of covariance structures. Psychological Bulletin, 88(3), 588-606. doi: 10.1037/0033-2909.88.3.588

Besen, E., Young, A. E., \& Shaw, W. S. (2015). Returning to work following low back pain: Towards a model of individual psychosocial factors. Journal of Occupational Rehabilitation, 25(1), 25-37.doi: 10.1007/s10926-014-9522-9

Borges, L. O., Silva, F. H. V. C., Melo, S. L., \& Martins, A. S. O. (2014). Socialização organizacional. Em Siqueira, M. M. M. (Ed.), Novas medidas do comportamento organizacional: ferramentas de diagnóstico e gestão (pp. 264-279). Porto Alegre: Artmed.

Byrne, B. M. (2001). Structural equation modeling with AMOS. Basic concepts, applications, and programming. Mahwah, NJ: Erlbaum.

Cardoso, H. F., \& Baptista, M. N. (2014). Escala de Autoeficácia no Trabalho (EAE-T): relatório técnico não publicado. Universidade São Francisco, Itatiba: São Paulo 
Chaudhary, R., Rangnekar, S., \& Barua, M. K. (2012). HRD climate, occupational self-efficacy and work engagement: A study from India. The Psychologist-Manager Journal, 15(2), 86-105. doi: 10.1080/10887156.2012.676938

Colombo, L., \& Zito, M. (2014). Demands, resources and the three dimensions of flow at work. A study among professional nurses. Open Journal of Nursing, 4, 255-264. doi: 10.4236/ojn.2014.44030.

Damásio, B. F. (2012). Uso da análise fatorial exploratória em Psicologia. Avaliação Psicológica, 11(2), 213-228.

Dancey, C. P., \& Reidy, J. (2006). Estatística sem matemática para psicologia usando SPSS para Windows (3a edição). Porto Alegre: Artmed.

Demo, G., Neiva, E. R., Nunes, I., \& Rozzett, K. (2014). Políticas e práticas de recursos humanos. Em Siqueira, M. M. M. (Ed.), Novas medidas do comportamento organizacional: ferramentas de diagnóstico e gestão (pp. 240-255). Porto Alegre: Artmed.

DePrince, A. P., Priebe, S. J., \& Newton, A. T. (2011). Learning about violence against women in research methods: A comparison to traditional pedagogy. Psychological Trauma: Theory, Research, Practice, and Policy, 3(3), 215-222.doi: 10.1037/a0024641

Erwins, C. J. (2001). The relationship of women's role strain to social support, role satisfaction, and self efficacy. Family Relations, 50(3), 230238. doi: 10.1111/j.1741-3729.2001.00230.x

Federici, R. A., \& Skaalvik, E. M. (2011). Principal self-efficacy and work engagement: Assessing a norwegian principal self-efficacy scale. Social Psychology of Education, 14(4), 575-600. doi: 10.1007/s11218-011-9160-4

Federici, R. A., \& Skaalvik, E. M. (2012). Principal self-efficacy: Relations with burnout, job satisfaction and motivation to quit. Social Psychology of Education, 15(3), 295-320. doi: 10.1007/s11218-012-9183-5

Ferrari, J. R. (2004). Australian eldercare providers: Comparing volunteers and temporary staff on work environment, interpersonal relationships, and self-efficacy. Evaluation \& the health professions, 27(4), 383-397. doi: 10.1177/0163278704270008

Ferreira, M. C., \& Assmar, E. M. L. (2008). Cultura organizacional. Em Siqueira, M. M. M. (Eds.), Medidas do comportamento organizacional: ferramentas de diagnóstico e gestão (pp. 125-138). Porto Alegre: Artmed.

Ferreira, M. C., \& Mendes, A. M. B. (2008). Contexto de trabalho. Em Siqueira, M. M. M. (Eds.), Medidas do comportamento organizacional: ferramentas de diagnóstico e gestão (pp. 111-124). Porto Alegre: Artmed.

Hair, J. F., Anderson, R. E., Tatham, R. L., \& Black, W. (2005). Análise multivariada de dados. Porto Alegre: Bookman.

Halbesleben, J. R. B. (2010). A meta-analysis of work engagement: Relationships with burnout, demands, resources and consequences. Em A. Bakker, \& M. P. Leiter (Eds.), Work engagement: A handbook of essential theory and research. (pp. 85-101). Hove and New York: Psychology Press.

Hennessy, K. D., \& Lent, R. W. (2008). Self-efficacy for managing work-family conflict validating the english language version of a hebrew scale. Journal of Career Assessment, 16(3), 370-383. doi: 10.1177/1069072708317383

Hu, L. T., \& Bentler, P. M. (1999). Cutoff criteria for fit indexes in covariance structure analysis: Conventional criteria versus new alternatives. Structural Equation Modeling, 6(1), 1-55. doi: 10.1080/10705519909540118

Huijs, J. J. J. M., Koppes, L. L. J., Taris, T. W., \& Blonk, R. W. B. (2012). Differences in Predictors of return to work among long-term sicklisted employees with different self-reported reasons for sick leave. Journal of Occupational Rehabilitation, 22(3), 301-311. doi: 10.1007/ s10926-011-9351-z

Lagervelda, S. E., Blonka, R. W. B., Brenninkmeijerb, V., \& Schaufelib, W. B. (2010). Return to work among employees with mental health problems: Development and validation of a self-efficacy questionnaire. Work \& Stress, 24(4), 359-375. doi: 10.1080/02678373.2010.532644

LeBlanc P., Schaufeli W. B., Salanova M., Llorens S., \& Nap, R. E. (2010) Efficacy beliefs predict collaborative practice among intensive care unit nurses. Journal of Advanced Nursing, 66(3), 583-594. doi: 10.1111/j.1365-2648.2009.05229.x

Lorenzo-Seva, U., Timmerman, M. E., \& Kiers, H.A.L. (2011). The Hull method for selecting the number of common factors. Multivariate Behavioral Research, 46(2), 340-364. doi: 10.1080/00273171.2011.564527.

Martins, M. C. F. (2008). Clima organizacional. Em Siqueira, M. M. M. (Eds.), Medidas do comportamento organizacional: ferramentas de diagnóstico e gestão (pp. 29-40). Porto Alegre: Artmed.

Munir F., \& Nielsen K. (2009) Does self-efficacy mediate the relationship between transformational leadership behaviours and healthcare workers' sleep quality? A longitudinal study. Journal of Advanced Nursing, 65(9), 1833-1843. doi: 10.1111/j.1365-2648.2009.05039.x

Muthén, L. K., \&Muthén, B. O. (2012). Mplus user's guide (7th ed.). Los Angeles, CA: Muthén \& Muthén.

O'Sullivan, D., Strauser, D. R., \& Wong, A. W. K. (2012). Five-factor model of personality, work behavior self-efficacy, and length of prior employment for individuals with disabilities: An exploratory analysis. Rehabilitation Counseling Bulletin, 55(3) 156-165. doi: $10.1177 / 0034355212437046$

Pajares, F. (1997). Current directions in self-efficacy research. Em M. L. Maehr, \& P. R. Pintrich (Eds.), Advances in motivation and achievement (vol. 10, pp. 1-49). Greenwich: JAI Press.

Pajares, F. (2002). Overview of social cognitive theory and self-efficacy. Recuperado de http://www.emory.edu/education/mfp/eff.html

Pasquali, L. (1999). Instrumentos psicológicos: Manual prático de elaboração. Brasília: LabPAM \& IBAPP

Pepe, S. J., Farnese, M. L., Avalone, F., \& Vecchione, M. (2010). Work self-efficacy scale and search forwork self-efficacy scale: A validation study in spanish and italian cultural contexts. Revista de Psicología del Trabajo y de las Organizaciones, 26(3), 201-210. Recuperado de http:// www.redalyc.org/pdf/2313/231316502004.pdf

Pereira, J. C. R. (1999). Análise de dados qualitativos: Estratégias metodológicas para as ciências da saúde, humanas e sociais. São Paulo: EDUSP/FAPESP

Piñgul, F. S. (2015). Measuring the impact of a supplemental civic education program on students' civic attitude and efficacy beliefs. Journal of Education and Training Studies, 3(2), 61-69. doi: 10.11114/jets.v3i2.600

Rauf, F. H. A. (2013). The role work family conflict self-efficacy ont the negative effect of organizational citizenship behavior. South East Asia Journal of Contemporary Business, Economics and Law, 3(1), 49-54. Recuperado de https://bit.ly/2xZGg6R

Reeb, R. N., Folger, S. F., Langsner, S., Ryan, C., \& Crouse, J. (2010). Self-Efficacy in Service-Learning Community Action Research: Theory, Research, and Practice. American Journal of Community Psychology, 46(3), 459-471. doi: 10.1007/s10464-010-9342-9.

Salanova, M., Lorente, L., Chambel, M. J., \& Martínez, I. M. (2011). Linking transformational leadership to nurses' extra-role performance: The mediating role of self-efficacy and work engagement. Journal of Advanced Nursing 67(10), 2256-2266. doi: 10.1111/j.1365-2648.2011.05652.x

Sistema de Avaliação de Testes Psicológicos - SATEPSI (2004). Avaliação dos testes psicológicos. Recuperado de http://www.pol.org.br. Acesso: 26/07/2015

Schyns, B., \& von Collani, G. (2002). A new occupational self-efficacy scale and its relation to per-sonality constructs and organisational variables. European Journal of Work and Organizational Psychology, 11(2), 219-241. doi: 10.1080/13594320244000148 
Shaw, W. S., Reme, S. E., Linton, S. J., \& Pransky, Y.H.G. (2011). $3^{\circ}$ place, PREMUS best paper competition: Development of the returntowork self-efficacy (RTWSE-19) questionnaire - psychometric properties and predictive validity. Scandinavian Journal of Work Environment \& Health, 37(2), 109-119. doi: 10.5271/sjweh.3139

Siqueira, M. M. M. (2008a). Medidas do comportamento organizacional: Ferramentas de diagnóstico e de gestão. Porto Alegre: Artmed.

Siqueira, M. M. M. (2008b). Satisfação no trabalho. Em Siqueira, M. M. M. (Ed.), Medidas do comportamento organizacional: ferramentas de diagnóstico e gestão (pp. 265-274). Porto Alegre: Artmed.

Siqueira, M. M. M. (2014). Novas medidas do comportamento organizacional: Ferramentas de diagnóstico e de gestão. Porto Alegre: Artmed.

Siqueira, M. M. M., Martins, M. C. F., Orengo, V., \& Souza, W. S. (2014). Engajamento no trabalho. Em Siqueira, M. M. M. (Ed.), Novas medidas do comportamento organizacional: ferramentas de diagnóstico e gestão (pp. 147-156). Porto Alegre: Artmed.

Siqueira, M. M. M., Orengo, V., \& Peiró, J. M. (2014). Bem-estar no trabalho. Em Siqueira, M. M. M. (Ed.), Novas medidas do comportamento organizacional: ferramentas de diagnóstico e gestão (pp. 39-51). Porto Alegre: Artmed.

Strauser, D. R., O'Sullivan, D., \& Wong, A. W. K. (2010). The relationship between contextual work behaviours self-efficacy and work personality: An exploratory analysis. Disability and Rehabilitation, 32(24), 1999-2008. doi: 10.3109/09638281003797380

Sweetman, D., \& Luthans, F. (2010). The power of positive psychology: Psychological capital and work engagement. Em A. Bakker, \& M. P. Leiter (Eds.), Work engagement: A handbook of essential theory and research. (pp. 39-53). Hove, New York: Psychology Press.

Thompson, B. (2004). Exploratory and confirmatory factor analysis: Understanding concepts and applications. Washington, DC: American Psychological Association.

Timmerman, M. E., \& Lorenzo-Seva, U. (2011). Dimensionality assessment of ordered polytomous items with parallel analysis. Psychological Methods, 16(2), 209-220. doi: 10.1037/a0023353.

Vax, S., Schreuer, N., \& Sachs, D. (2012). Work-related self-efficacy of occupational therapists in mental health. Scandinavian Journal of Occupational Therapy, 19(1), 42-48. doi: 10.3109/11038128.2010.527366

Velicer, W. F. (1976). Determining the number of components from the matrix of partial correlations. Psychometrika, 41(3), $321-327$. doi:10.1007/BF02293557

Vinokur, A., \& Schul, J. (2002). The web of coping resources and pathways to reemployment following a job loss. Journal of Occupational Health Psychology, 7(1), 68-83. doi: 10.1037//1076-8998.7.1.68

Xanthopoulou, D., Bakker, A. B., Demerouti, E., \& Schaufeli,W. B. (2007). The role of personal resources in the job demands-resources model. International Journal of Stress Management, 14(2), 121-141. doi: 10.1037/1072-5245.14.2.121

Waghorn, G., Chant, D., \& King, R. (2005). Work-related self-efficacy among community residents with psychiatric disabilities. Psychiatric Rehabilitation Journal, 29(2), 105-113. doi: 10.2975/29.2005.105.113

\section{Sobre os autores}

Hugo Ferrari Cardoso é psicólogo, pós-doutorado pela Universidade São Francisco. Atualmente é docente assistente do programa de pós-graduação e graduação da Universidade Estadual Paulista "Júlio de Mesquita Filho" (UNESP/Bauru); Bauru/SP.

Makilim Nunes Baptista é psicólogo, doutor em Psicologia pela Universidade Federal de São Paulo. Atualmente é docente do programa de pós-graduação e graduação da Universidade São Francisco (USF); Campinas/SP. 